\title{
Flow and heat transfer of aligned magnetic field with Newtonian heating boundary condition
}

\author{
A R M Kasim* ${ }^{*}, N S$ Arifin, $S M$ Zokri, and $M Z$ Salleh \\ Applied \& Industrial Mathematics Research Group, Faculty of Industrial Sciences \& Technology, \\ Universiti Malaysia Pahang, Malaysia
}

\begin{abstract}
Influence of aligned magnetic field on the steady boundary layer flow and heat transfer over a stretching sheet with Newtonian heating boundary condition is considered. The transformed governing nonlinear boundary layer equations in the form of ordinary differential equations are solved numerically by Keller box method. The details on computation have been presented and elaborated. The obtained numerical solutions have been captured graphically in the form of velocity and temperature distributions for different values of aligned angle, magnetic field parameter, Prandtl number and conjugate parameter. It is found that, increases in aligned angle associated with magnetic field delayed the velocity profile of the flow and enhances the temperature profile.
\end{abstract}

\section{Introduction}

The study of the boundary layer flow and heat transfer over a stretching sheet in a viscous incompressible fluid has gained considerable attention for its vital roles in industrial process of cooling of metallic plates in a cooling bath, polymer and metal sheet, paper production, metal wires and etc. Essentially, the rate of heat transfer and skin friction coefficient are significant in determines the quality of the final product at the stretching surface. In view of this, Sakiadis [1] is a pioneer who worked on boundary layer flow of Newtonian fluid induced by a continuous moving plate at uniform speed from a slit into a fluid at rest. It is interesting knowledge since he obtained the mathematical features which represent the properties of the flow problem. Later, Sakiadis's theoretical predictions were verified experimentally by Tsou et al. [2]. Subsequently, the similar study has been extended by many authors to temperature distribution for the case of stretching sheet [3-5].

The thermal boundary conditions have a great influence on the heat transfer in laminar boundary layer flow problem. Most of the literatures are for the fluid flow on stretched surface which is driven by prescribed surface temperature (PSF) or prescribed surface heat flux (PHF) [6-8]. However, there are other situations in which the heat transfer rate is proportional to the local difference in temperature with ambient conditions or usually termed as conjugate conditions [9] and also Newtonian Heating [10]. Newtonian Heating $(\mathrm{NH})$ is practically applied in engineering devices, for instance the heat exchanger which transferring heat from one medium to another. Thus, the effects of $\mathrm{NH}$ on the temperature distribution for laminar boundary layer flow were highlighted by several authors [11-15].

* Corresponding author: rahmanmohd@ump.edu.my 
The involving of magnetohydrodynamic (MHD) can be found in many engineering applications such as MHD generator, nuclear reactor for liquid-metal cooling and electromagnetic casting. The momentum and heat transfer in the boundary layer flow for various fluids and geometry surface are controlled by applied magnetic field. In the metallurgical processes, the strip or filaments is continuously forced to pass through a quiescent fluid for cooling and occasionally the strip may stretch throughout the process[16]. Although the existing literature has been carried out on the transverse magnetic field flow problems, several researchers have shown an increased interest in investigating the effect of applied magnetic field at any angle in the range of $0^{\circ}-90^{\circ}$ on the flow region. For that, a mathematical analysis of aligned magnetic field on boundary layer flow for non-Newtonian fluid has been published [17-19].

In the present paper, the influence of aligned magnetic field and Newtonian heating on the steady flow of viscous fluid over a stretching sheet is considered. The numerical algorithm of Keller box method is developed in Matlab software to compute the problem and the numerical findings of the flow model are displayed in graphical form.

\section{Problem formulation}

The two dimensional steady flow of incompressible and electrically conducting viscous fluid over a stretching sheet with Newtonian heating $(\mathrm{NH})$ is considered. The velocity of the stretching surface is $u_{w}(x)$ with $a$ being a positive constant and an acute angle $\alpha_{1}$ with aligned magnetic field is induced to the flow as shown in figure 1.

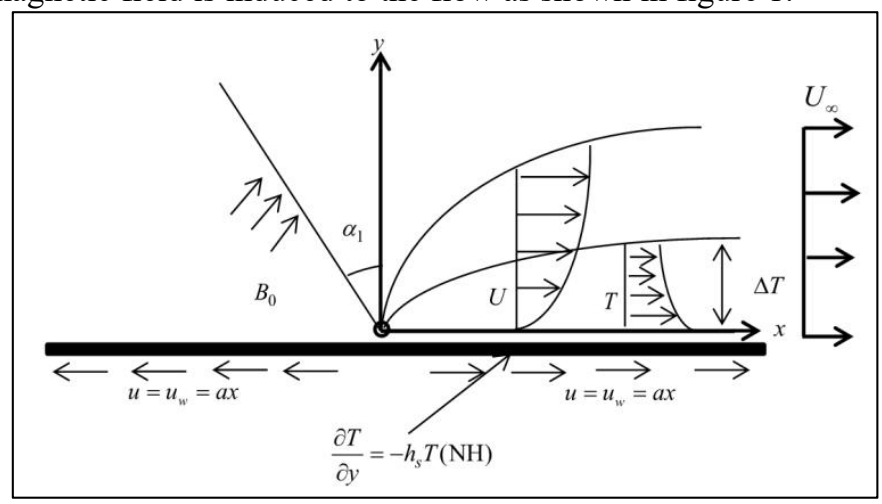

Fig.1. Flow configuration.

Under the boundary layer and Boussinesq approximations, the governing equations of the flow can be written as ([20])

$$
\begin{gathered}
\frac{\partial u}{\partial x}+\frac{\partial v}{\partial y}=0 \\
u \frac{\partial u}{\partial x}+v \frac{\partial u}{\partial y}=v\left(\frac{\partial^{2} u}{\partial y^{2}}\right)-\frac{\sigma}{\rho} u B_{0}{ }^{2} \sin ^{2} \alpha_{1} u=u_{w}(x)=a x, \quad v=0, \frac{\partial T}{\partial y}=-h_{s} T(\mathrm{NH}) \\
u \frac{\partial T}{\partial x}+v \frac{\partial T}{\partial y}=\alpha\left(\frac{\partial^{2} T}{\partial y^{2}}\right)
\end{gathered}
$$

with the boundary conditions

at $y=0$

$$
u \rightarrow 0, T \rightarrow T_{\infty} \quad \text { at } y \rightarrow \infty
$$


where $u_{w}(x)$ is the velocity of the stretching surface with $a$ being a positive constant, $\alpha_{1}$ is the inclined angle, $\sigma$ is electrical conductivity, $h_{s}$ is the heat transfer parameter, $T$ is the fluid temperature, $T_{\infty}$ is the ambient temperature, $v$ is the kinematic viscosity, $\alpha$ is the thermal diffusivity, $B_{0}$ is the magnetic-field strength.

Introducing the similarity transformation on (1)-(4),

$$
\eta=\left(\frac{a}{v}\right)^{1 / 2} y, \quad \psi=(a v)^{1 / 2} f(\eta), \quad \theta(\eta)=\frac{T-T_{\infty}}{T_{\infty}}
$$

where $\psi$ is the stream function defined as $u=\frac{\partial \psi}{\partial y}$ and $v=-\frac{\partial \psi}{\partial x}$, yield

$$
\begin{gathered}
f^{\prime \prime \prime}(\eta)+f(\eta) f^{\prime}(\eta)-f^{\prime 2}(\eta)-M f^{\prime}(\eta) \sin ^{2} \alpha_{1}=0 \\
\frac{1}{\operatorname{Pr}} \theta^{\prime \prime}(\eta)+f \theta^{\prime}(\eta)=0
\end{gathered}
$$

and boundary conditions becomes

$$
\begin{aligned}
& f(0)=0, f^{\prime}(0)=1, \theta^{\prime}(0)=-\gamma[1+\theta(0)] \text { at } \eta=0 \\
& f^{\prime}(\eta) \rightarrow 0, \quad \theta(\eta) \rightarrow 0 \quad \text { as } \eta \rightarrow \infty
\end{aligned}
$$

where $M=\sigma B_{0}^{2} / \rho a$ is the magnetic field parameter, $\operatorname{Pr}=v / \alpha$ is the Prandtl number and $\gamma=h_{s}(v / a)^{1 / 2}$ is the conjugate parameter for Newtonian heating.

The non-dimensional quantities of physical interest in this problem are the skin friction coefficient $C_{f}$ and the local Nusselt number $N u_{x}$ are defined by

$$
C_{f}=\frac{\tau_{w}}{(1 / 2) \rho U^{2}(x)}, \quad N u_{x}=\frac{x q_{w}}{k\left(T_{w}-T_{\infty}\right)}
$$

where $\tau_{w}$ is the rate of heat transfer, $q_{w}$ is the surface heat flux and $\rho$ is the fluid density which are defined as

$$
\tau_{w}=\mu\left(\frac{\partial u}{\partial y}\right)_{y=0}, \quad q_{w}=-k\left(\frac{\partial T}{\partial y}\right)_{y=0}
$$

With $\mu=\rho v$ and $k$ are dynamic viscosity and thermal conductivity respectively. Substituting (10) into (9) and using (5), yield

$$
C_{f} \operatorname{Re}_{x}^{1 / 2}=f^{\prime \prime}(0), \quad N u_{x} \operatorname{Re}_{x}^{-1 / 2}=\gamma\left(1+\frac{1}{\theta(0)}\right)
$$

where $\operatorname{Re}_{x}=\left(a x^{2} / v\right)$ is the Reynolds number.

\section{Methodology}

The transformed ordinary differential equations (6) and (7) with the boundary conditions (8) are solved using Keller-box method. The method is started by transforming the nonlinear governing differential equations into first order system. Then, the first order system is approximated using central difference. Since the system is nonlinear equation, the Newton's method is applied to linearize the system and finally, the solutions can be solved by block elimination technique. The calculations are executed in program using Matlab.

The step size of time and space can be arbitrary since this method is implicit with second order accuracy which makes it suitable to solve the parabolic partial differential 
equations efficiently [21]. However, the computation could be time consuming if the small step size of time and space is inserted. From the analysis on computational work, the values of $\eta_{\infty}=3-10$ and uniform grid of $\Delta \eta=0.02$ provide the outcome on the velocity and temperature profiles which asymptotically fulfilled the boundary conditions (8) as depicted in figures $2-5$.

\section{Results and discussion}

The present results presented in this paper are for various values of non-dimensionless parameter at the fixed values of $\operatorname{Pr}=7, \gamma=0.1, \alpha_{1}=\pi / 6$ and $M=2$. The influences of non dimensionless parameter, specifically aligned angle $\alpha_{1}$, magnetic field parameter $M$, Prandtl number $\operatorname{Pr}$ and conjugate parameter $\gamma$ are given in table and graph. For validation, the present numerical results are compare with the previous existed results has been made (see Table 1$)$ by neglecting the magnetic field $(M=0)$ or aligned angle $\left(\alpha_{1}=0\right)$ and $\gamma=1$. A good agreement of the present results is observed with those previous results from table 1 . Therefore, it is assured that a high accuracy in the present numerical results.

Table 1. Comparative results for $\theta(0)$.

\begin{tabular}{|c|c|c|c|}
\hline $\operatorname{Pr}$ & Salleh et al.[11] & Turkyilmazoglu [22] & Present \\
\hline 3 & 6.02577 & 6.05159 & 6.02578 \\
\hline 5 & 1.76594 & 1.76040 & 1.76594 \\
\hline 7 & 1.13511 & 1.11681 & 1.13511 \\
\hline 10 & 0.76531 & 0.76452 & 0.76531 \\
\hline
\end{tabular}

Figures 2 and 3 illustrated the effects of aligned angle $\alpha_{1}$ on distribution of velocity and temperature of fluid respectively. It is clearly shown in those figures that increasing value of $\alpha_{1}$ is to decrease values of fluid's velocity and increase the fluid's temperature profile. Physically, an increase values of $\alpha_{1}$ strengthens the magnetic field which acts opposite to the flow region and thereby enhances the Lorentz force that resulted to decelerate fluid flow.

Figure 4 displayed the influence of $\operatorname{Pr}$ on temperature profile. From the figure, it is observed that increase in $\operatorname{Pr}$ decreases the temperature profile. The viscous diffusion rate dominates the thermal diffusion rate the viscous diffusion rate for a large value of $\mathrm{Pr}$. Therefore, a raise of $\operatorname{Pr}$ decreases the thermal conductivity and the heat transfer will be less convective which causes the thermal boundary layer thickness become thinner. Figure 5 elucidates the effect of conjugate parameter $\gamma$ on temperature profile. The temperature profile increases significantly with an increase in $\gamma$. As expected, the increment in $\gamma$ lead to increase in heat transfer parameter $h_{s}$, thereby increase in temperature distribution.

Figures 6 and 7 depicted the combination effect of magnetic field and aligned angle on the skin friction coefficient and Nusselt number. It is noticed from those figures that a rise in magnetic field and aligned angle reduces the skin friction coefficient and Nusselt number. Consequently, the temperature of the aligned angle decrease as exhibited in Figure 3. This can be explained mathematically based on equation (11). 


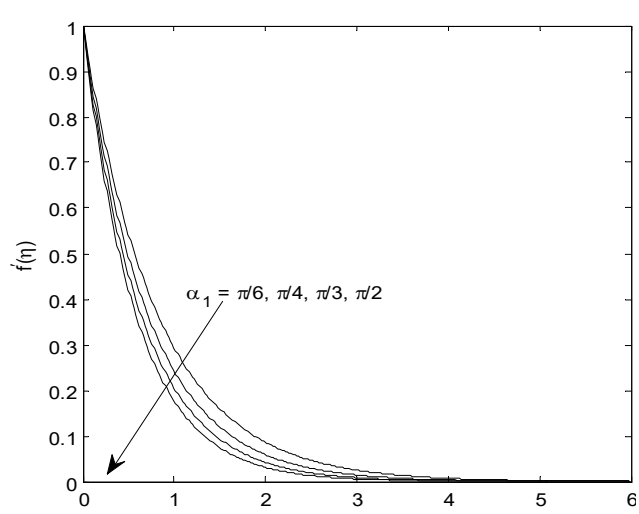

Fig. 2. Velocity profile for ${ }^{\eta}$ different values of $\alpha_{1}$

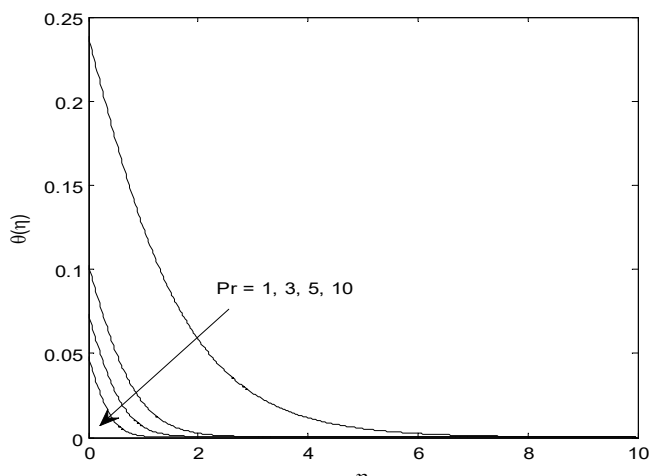

Fig. 4. Temperature profile for different values of $\operatorname{Pr}$.

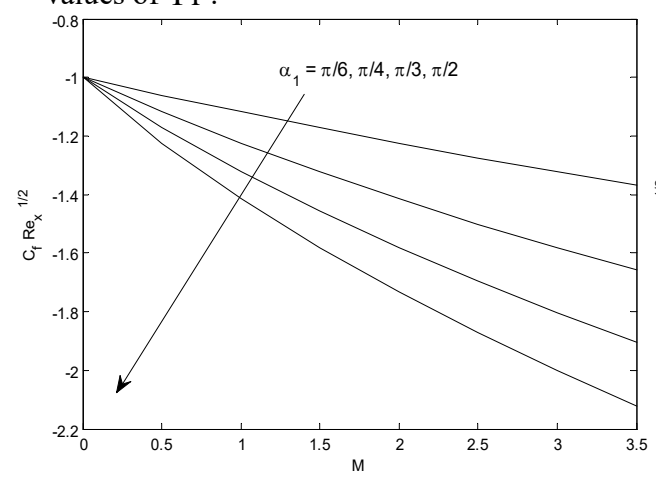

Fig. 6. Variation of $M$ and $\alpha_{1}$ on the skin friction coefficient.

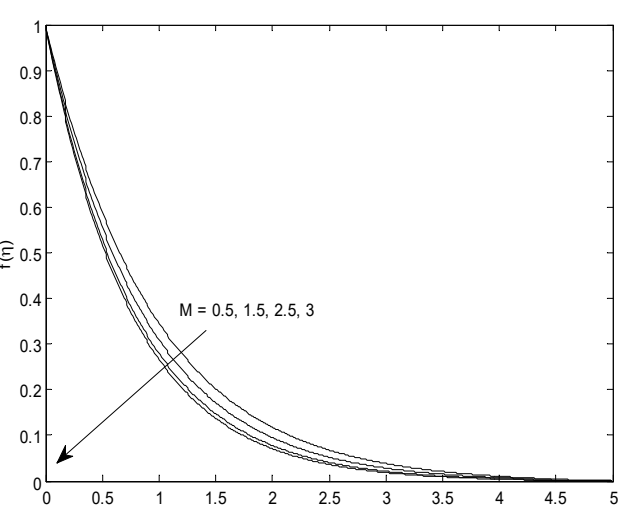

Fig. 3. Temperature profille for different values of

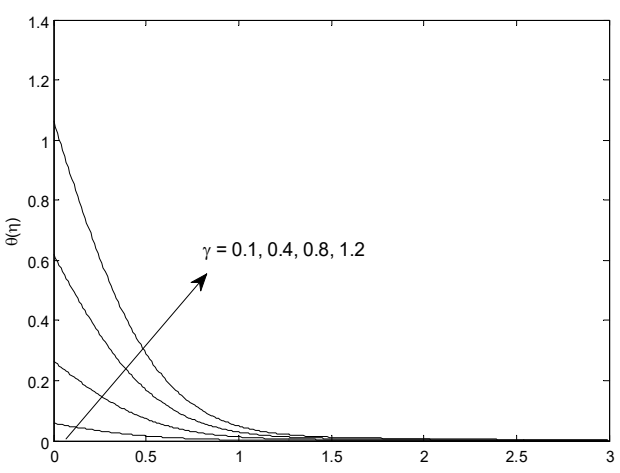

Fig. 5. Temperature profile for different

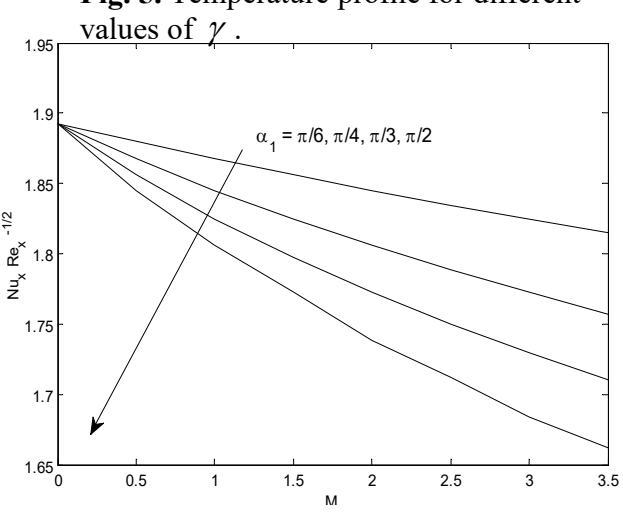

Fig. 7. Variation of $M$ and $\alpha_{1}$ on the Nusselt number.

\section{Conclusion}

The numerical solutions on the flow of aligned magnetic field and heat transfer over a stretching sheet fluid with Newtonian heating is investigated. The increment of aligned angle is to strengthen the magnetic field which led the fluid velocity to decrease and increase temperature profile. The increasing Prandtl number reduces the temperature profile. Meanwhile, the conjugate parameter increases with the temperature profile. 


\section{Acknowledgments}

The authors gratefully acknowledge the financial support received from Universiti Malaysia Pahang for (PGRS170397, RDU 160330 \& RDU 170328).

\section{References}

1. Sakiadis B 1961 AIChE J. 7 26-28

2. Tsou F, Sparrow E and Goldstein R J 1967 Int. J. Heat Mass Transfer 10 219-235

3. Dutta B, Roy P and Gupta A 1985 Int. Comm. Heat Mass Transfer 12 89-94

4. Grubka L and Bobba K 1985 J. Heat Transfer 107 248-250

5. Char M-I 1988 J. Math. Anal. Appl. 135 568-580

6. Kasim A R M, Othman Z S, Shafie S and Pop I 2013 Int. J. Numer. Methods Heat Fluid Flow 23 1242-55

7. Aurangzaib, Kasim A R M, Mohammad N F and Sharidan S 2013 J Appl Sci Eng 16 141-150

8. Salleh M Z and Nazar R M 2008 J. Quality Measur. Anal. 4 57-69

9. Merkin J and Pop I 1996 Int. J. Heat Mass Transfer 39 1527-1534

10. Merkin J 1994 Int. J. Heat Fluid Flow 15 392-398

11. Salleh M, Nazar R and Pop I 2010 J. Taiwan Inst. Chem. Eng. 41 651-655

12. Arifin N S, Zokri S M, Kasim A R M, Salleh M Z and Mohammad N F 2016 The National Conference for Postgraduate Research 2016 p 266-274

13. Kasim A R M, Mohammad N F, Anwar I and Shafie S 2013 Recent Adv. Mat. 4 182-9

14. Kasim A R M, Mohammad NF, Aurangzaib Sharidan S 2012 World Acad. Sci. Eng. Technol. 6 628-633

15. Al-Sharifi H A M, Kasim A R M, Aziz L A, Shafie S 2017 Indian J. Sci. Technol. 10 $1-5$

16. Vajravelu K and Nayfeh J 1992 Int. J. Non Linear Mech. 27 937-945

17. Aziz L A, Kasim A R M, Al-Sharifi, H A M, Salleh, M Z, Mohammad N F, Shafie S and Ali A 2017 AIP Conf. Proc. vol 1842 p 030005

18. Sulochana C, Sandeep N, Sugunamma V and Kumar B R 2015 Appl. Nanosc. 6 737746

19. Sandeep N, Raju C S K, Sulochana C and Sugunamma V 2015 Internat. J. Sci. Eng. 8 151-8

20. Arifin N S, Zokri S M, Aziz L A, Kasim A R M, Salleh M Z and Mohammad NF 2017 AIP Conf. Proc. vol 1842 p 030006

21. Al-Shibani F, Ismail A M and Abdullah F 2012 Journal of Applied Mathematics and Bioformatics 2 69-84

22. Turkyilmazoglu M 2016 Int. J. Non Linear Mech. 83 59-64 